\title{
The Relationship Between Tumor Glucose Metabolism and Host Systemic Inflammatory Responses in Patients with Cancer: A Systematic Review
}

\author{
Ross D. Dolan ${ }^{1}$, Naomi G. McLees ${ }^{1}$, Ahmer Irfan ${ }^{1}$, Stephen T. McSorley ${ }^{1}$, Paul G. Horgan ${ }^{1}$, David Colville ${ }^{2}$, \\ and Donald C. McMillan² \\ ${ }^{1}$ Academic Unit of Surgery, School of Medicine, University of Glasgow, Glasgow Royal Infirmary, Glasgow, United Kingdom; and \\ ${ }^{2}$ West of Scotland PET Centre, Gartnavel Hospital, Glasgow, United Kingdom
}

One of the most important and long recognized characteristics of tumor cells is their dysregulated cellular energetics with anaerobic driven glucose uptake. In patients with cancer, the prognostic value of the systemic inflammatory response has been well established, and the recent combination of PET and CT scanning combines the assessment of tumor physiologic activity with detailed anatomic localization. The aim of this study was to perform a systematic review of the assessment of the relationship between both the tumor and the host inflammatory responses using PET/CT. Methods: An extensive literature review using targeted subject headings was performed in the U.S. National Library of Medicine, the Excerpta Medica database, and the Cochrane Database of Systematic Reviews on March 31, 2018. On completion of the online search, the title and abstracts of each identified study were examined for relevance. Studies with duplicate datasets, not available in English, and that did not have full text availability were excluded. Full texts of relevant articles were obtained and were then examined to identify any further relevant articles. Results: Twelve studies containing 2,588 patients were included in the final analysis. All of the included studies used the ${ }^{18} \mathrm{~F}-\mathrm{FDG}$ tracer in PET/CT imaging and had biochemical assessment of the systemic inflammatory response. Most studies showed a direct relationship between the tumor and bone marrow glucose uptake and host systemic inflammatory responses as measured by C-reactive protein $(n=2)$, albumin $(n=2)$, white cell count $(n=3)$, neutrophils $(n=2)$, and platelets $(n=2)$. Most of the studies $(n=8)$ also showed a direct relationship between tumor and bone marrow glucose uptake and poor outcomes. Conclusion: This review suggests a direct relationship between the tumor and bone marrow glucose uptake and host systemic inflammation. This may suggest new approaches for more optimal therapeutic targeting and monitoring strategies in patients with cancer.

Key Words: cancer; glucose; positron emission tomography/computed tomography; systemic inflammation markers; survival

J Nucl Med 2019; 60:467-471

DOI: 10.2967/jnumed.118.216697

\footnotetext{
Received Jun. 26, 2018; revision accepted Aug. 22, 2018.

For correspondence or reprints contact: Ross D. Dolan, Academic Unit of Surgery, University of Glasgow, New Lister Building, Glasgow Royal Infirmary, Glasgow, G4 OSF, United Kingdom.

E-mail: Ross.Dolan@glasgow.ac.uk

Published online Aug. 30, 2018.

COPYRIGHT (C) 2019 by the Society of Nuclear Medicine and Molecular Imaging.
}

C ancer remains one of the leading causes of mortality worldwide and is responsible for approximately 8.8 million deaths per year (1). Overall, it has been estimated that 1 in 3 people will develop cancer in their lifetime, and 1 in 4 will die from it $(2,3)$. Indeed, in the United Kingdom alone it is estimated that 150,000 people die of cancer each year $(1,3)$. Four cancers-lung, colorectal, breast, and prostate-account for approximately half of all new cases and deaths (2).

At a cellular level, there are several traits of cancer that define its malignancy. These include genome instability, limitless replicative potential, self-sufficiency in growth signals, insensitivity to antigrowth signals, the ability to evade apoptosis, sustained angiogenesis, tissue invasion and metastasis, abnormal metabolic pathways, inflammation, and evasion of the immune system $(4,5)$. All these hallmarks create what is known as the tumor microenvironment (TME (4-6)). The TME is composed of heterogeneous cell populations including tumor cells, immune cells, fibroblasts, adipocytes, blood vessels, and the extracellular matrix. Therefore, there are interactions between malignant and nontransformed cells via a host of signaling molecules) (7). The tumor and its environment are constantly interacting and this is an integral part of the tumor physiology, structure, and function. The relationship between the tumor and its environment is essential to promote tumor cell growth and the development of metastasis (8).

An important and long recognized characteristic of tumor cells is the dysregulated cellular energetics that results in the increased uptake of glucose (9). Warburg observed that tumor cells predominately produced adenosine $5^{\prime}$-triphosphate via a high rate of glycolysis and consumption of glucose via the conversion of glucose to lactic acid. He recognized that this was inefficient for the tumor cell to produce adenosine 5'-triphosphate when compared with normal oxidative phosphorylation $(9,10)$. Moreover, because of this anaerobic glycolysis and lactic acid formation the TME would become acidic, allowing for the de-differentiation of normal and malignant cells (11). Warburg hypothesized that this metabolic defect was the basis of tumor formation. In recent years it has been concluded that this metabolic defect is the result of genetic damage. Nevertheless, the impact of such dysregulated energetics of the tumor cell remains of considerable interest.

The TME is likely to have a direct impact on the innate immune response and activation of the systemic inflammatory response (SIR). This can be evidenced by increases in the circulating acute 
phase proteins such as C-reactive protein (CRP) and albumin and innate immune cells such as neutrophils and monocytes (12). These immune cells are also metabolically active, requiring large amounts of glucose.

The prognostic value of the CRP, albumin, and neutrophil counts in cancer has been well established in observational studies $(13,14)$. In the last $15 \mathrm{y}$, there has been a movement toward the use of combined prognostic scores such as the Glasgow Prognostic Score (GPS)/modified GPS (CRP and albumin) and ratios such as the NLR (neutrophils and lymphocytes) to standardize and maximize prognostic value $(15,16)$.

Therefore, it is of interest that imaging studies of the tumor have become an important element in the evaluation of detecting, staging, and management of patients with cancer (17). PET is an established nuclear imaging technique based on the uptake of glucose that can examine the metabolism of tumors. However, PET provides relatively poor anatomic information whereas CT is commonly used in the initial diagnosis and staging of cancers.

The recent routine clinical combination of PET and CT gives anatomic information with associated assessment of tumor physiologic activity (18). This provides better identification of metabolically active lesions, improving the diagnostic accuracy and localization of both the primary and the metastatic lesions. In the oncologic setting, the tracer ${ }^{18} \mathrm{~F}-\mathrm{FDG}$ is commonly used due to its longer half-life, which aids in transportation and clinical application (19). However, a disadvantage of this tracer is that it is not tumor cell-specific and can accumulate where there are metabolically active cells such as immune cells. For example, it is recognized to accumulate in bone marrow (BM), presumably due to formation of metabolically active immune cells. This additional variability that can occur with uptake parameters such as the SUV (which depends on appropriate calibration and reconstruction methods with intersite variability and on lesion or organ segmentation) has resulted in normalizing uptake to other metabolically active tissues. Interestingly, an elevated BM-to-liver ratio has been reported to have prognostic value in a variety of common solid tumors and an increased cytokine load due to malignancy (20).

On the basis of the above, it is hypothesized that glucose metabolism in both tumor and host inflammatory responses are related. This present review is timely given the rapidly expanding role of immune therapies (e.g., immune checkpoint inhibition and adoptive T-cell therapy) to treat patients with metastatic cancers. Therefore, the aim of this study was to perform a systematic review of the relationship between tumor and host inflammatory glucose metabolism using PET/CT. A better understanding of these processes would be useful to inform therapeutic strategies for patients with cancer.

\section{MATERIALS AND METHODS}

This systematic review of published literature was undertaken according to a predefined protocol described in the PRISMA-P statement. The primary outcome of interest of this systematic review was the relationship between tumor and host inflammatory glucose metabolism, specifically using PET/CT imaging in patients with cancer. The secondary outcome of interest of this systematic review was the association between tumor and host inflammatory glucose metabolism as measured by PET/CT imaging and survival in patients with cancer.

Studies were identified via a literature search of the electronic databases the U.S. National Library of Medicine, the Excerpta Medica database, and the Cochrane Database of Systematic Reviews between
1984 and 2018 using the following key words: cancer, malignancy, metastasis, inflammation, glucose, positron, CT and PET/CT (last search update on March 31, 2018).

To be eligible for inclusion, studies had to meet the following criteria: patients with cancer; PET/CT analysis the imaging modality used; tumor or BM activity measured by either $\mathrm{SUV}_{\max }, \mathrm{SUV}_{\text {mean }}$, or the BM-to-liver ratio (BLR is the mean BMSUV-to-mean liver SUV ratio); and markers of the systemic inflammatory response in the form of acute phase proteins (CRP and albumin) or components of the differential blood cell counts (neutrophils, leukocytes, monocytes, and platelets) and their composite scores such as the mGPS, platelet lymphocyte ratio (PLR), and NLR. Exclusion criteria included studies not performed in patients with cancer; studies not using PET/CT as the main imaging modality; studies not assessing tumor and BM activity; and studies not including measurement of the SIR.

On completion of the online search, the title and abstract of each identified study was examined for relevance. Studies not in cancer patients, studies not available in English, and those published in abstract form only were excluded. Where there were multiple publications from the same cohort, the most recent paper was included. Full texts were obtained for all studies deemed potentially relevant. Once further exclusions outlined above were performed, the bibliographies of all included articles were subsequently hand searched to identify any additional studies. Due to the small number of studies and the heterogeneity of tumor type and tumor/ BM activity assessment, metaanalysis was not performed.

\section{RESULTS}

\section{Study Selection Process}

The study selection process is summarized in Figure 1. Initial search strategy identified 207 articles whose titles and abstracts were reviewed. Articles were excluded if they had not been performed in humans $(n=64)$, no full texts were available $(n=12)$, were a systematic review/metaanalysis $(n=32)$, and they were not published in English $(n=6)$. This led to a review of the full text of 93 articles. A further 83 articles were excluded as there was no direct comparison between the SIR and PET/CT output. The remaining 10 articles had their bibliographies reviewed in a systematic manner. This identified a further 2 articles to be included in the final analysis, leading to final figure of 12 articles considered in the present systematic review (20-31).

\section{Overall Analysis}

The 12 included studies contained a total of 2,453 patients with the number of patients included in individual studies varying from 32 to 1,034 (Supplemental Table 1; supplemental materials are available at http://jnm.snmjournals.org). There was a wide variety in cancer anatomic locations including lung $(n=4)$, oral $(n=3)$, colorectal $(n=2)$, gastric $(n=1)$, head and neck $(n=1)$, and multiple anatomic locations $(n=1)$. Geographically, studies were from Korea $(n=5)$, China $(n=2)$, Belgium $(n=1)$, Taiwan $(n=1)$, Canada $(n=1)$, Japan $(n=1)$, and the United Kingdom $(n=1)$.

Most studies showed a direct relationship between the host systemic inflammatory response and the indices of ${ }^{18} \mathrm{~F}-\mathrm{FDG}$ accumulation as measured by BLR $(n=5)$, BMSUVmax $(n=4)$, tumor (T) SUVmax $(n=4)$, BMSUVmean $(n=2)$, nodal disease (N) SUVmax $(n=2), \operatorname{SUV}_{\text {peak }}(n=1)$, metabolic tumor volume $(n=1)$, and total lesion glycolysis $(n=1)$. In addition, most studies showed a direct relationship between survival and indices of ${ }^{18} \mathrm{~F}-\mathrm{FDG}$ accumulation BLR $(n=4)$, TSUVmax $(n=3)$, BMSUVmean $(n=2)$, BMSUVmax $(n=1)$, NSUVmax $(n=1)$, and total lesion glycolysis $(n=1)$. 


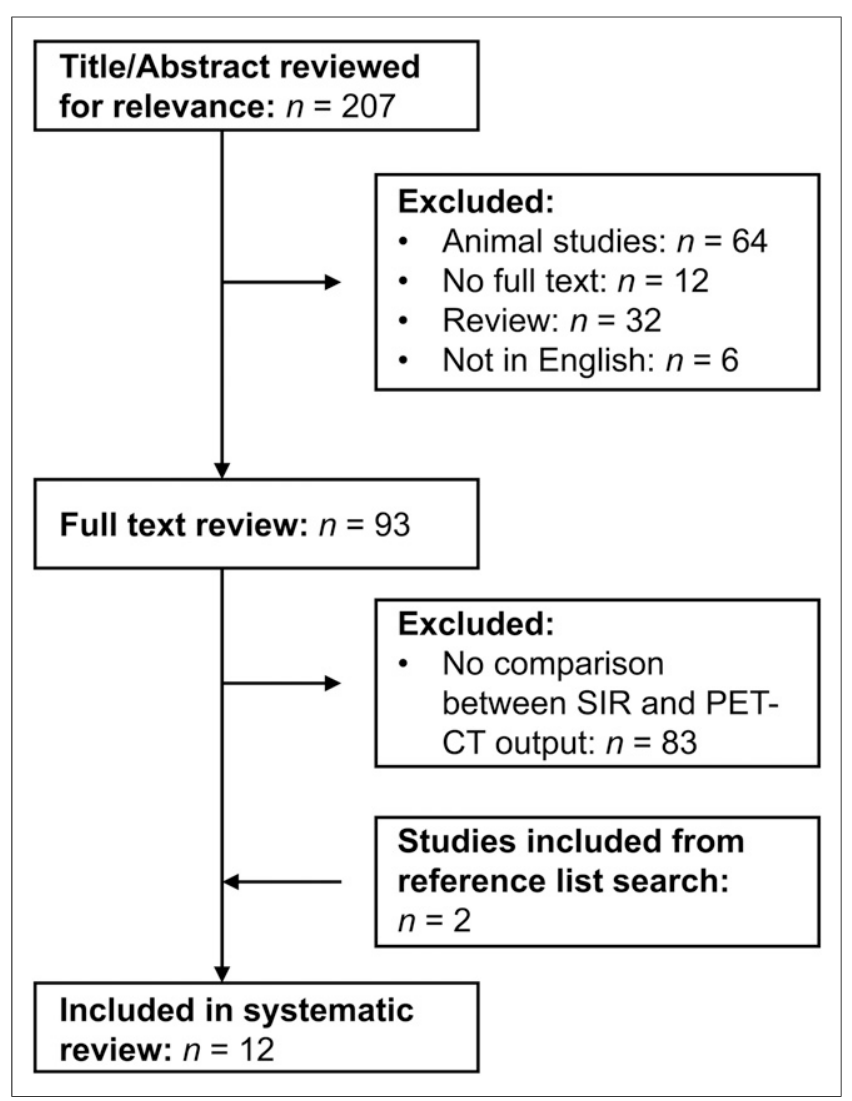

FIGURE 1. A PRISMA flowchart demonstrating study selection process.

All studies used the radioisotope ${ }^{18}$ F-FDG. There was some variation in the type of scanners used, with the most common scanners being Siemens $(n=5)$ and GE Healthcare $(n=4)$. In all studies, patients were required to fast for a minimum of $4-6 \mathrm{~h}$ before the PET/CT study protocol was initiated and fasting blood glucose levels were measured before the administration of ${ }^{18} \mathrm{~F}$ FDG. Most studies had a blood glucose threshold level of less than $150.0 \mathrm{mg} / \mathrm{dL}$ for the injection of the radioisotope. There was some variation in the activity of ${ }^{18} \mathrm{~F}-\mathrm{FDG}$ administered, however, all studies used weight-based protocols with administered activities ranging between 230 and $555 \mathrm{MBq}$. PET acquisition in most studies was from the base of the skull to the proximal thigh, using $6-8$ bed positions, acquired $60 \mathrm{~min}$ after ${ }^{18} \mathrm{~F}-\mathrm{FDG}$ administration. All reconstructions involved CT attenuation correction and iterative reconstruction algorithms specific to the camera manufacturer's software. Regions of interest were either drawn freehand, using a minimum SUV cutoff or using isocontour software. The SUV parameters measured varied slightly although in general the maximum and $\mathrm{SUV}_{\text {mean }}$ values were measured for the primary tumor (TSUVmax, TSUVmean), nodal disease (NSUVmax, NSUVmean), and BM (BMSUVmax, BMSUVmean). The BM-toliver ratio (BLR) was defined using $S U V_{\text {mean }}$ measurements in the $\mathrm{BM}$, obtained mainly from vertebral bodies, and $\mathrm{SUV}_{\text {mean }}$ from a region of interest in the right lobe of liver.

Most studies focused on patients with stage I-III disease who were treated with surgical resection with or without adjuvant chemoradiotherapy $(n=8)$. In those studies in which surgery was not the mainstay of treatment, only 1 study had a majority of metastatic disease (79.2\%) (24). Two studies were in ear nose and throat
(ENT) cancers, with the treatment of choice being concurrent chemoradiotherapy and definitive radiotherapy $(24,26)$. One study was in patients with advanced non-small cell lung cancer (NSCLC) not amenable to surgical resection, and 1 study was in multiple cancer types again not amenable to surgical resection $(20,28)$.

Most studies used singular markers of the systemic inflammatory response including the white cell count $(n=9)$, CRP $(n=7)$, hemoglobin $(n=4)$, albumin $(n=3)$, neutrophils $(n=2)$, platelets $(n=2)$, lymphocytes $(n=1)$, and monocytes $(n=1)$. In addition composite ratios and scores were used in several studies including the NLR $(n=6)$, PLR $(n=4)$, and mGPS $(n=1)$. Multiple markers of the systemic inflammatory response were used; however, there was considerable heterogeneity in the specific markers used.

Therefore, a metaanalysis could not be meaningfully performed due to the heterogeneity of tumor stage, tumor type, and markers of the SIR.

\section{Relationship Between Tumor Glucose Metabolism Using TSUVmax/mean, BMSUVmax/mean, and BLR and Host Inflammatory Responses}

As can be seen in Supplemental Table 1, most studies reported a significant association between activation of the SIR and increased tumor, BM, and nodal uptake of ${ }^{18} \mathrm{~F}-\mathrm{FDG}$ on PET/CT. In particular, the largest study $(n=1,034)$ included in this review reported such a relationship (25).

Jeong et al. compared the prognostic values of circulating blood cell-based parameters and tumor ${ }^{18} \mathrm{~F}$-FDG uptake in patients with stage I NSCLC (25). In total, 1,034 patients were included in this study. They were all newly diagnosed with NSCLC and underwent PET/CT scanning as part of their preoperative workup before undergoing surgical resection (25). Biochemical and hematologic measurements in the form of white cell count, neutrophil, lymphocyte, and platelet counts were taken (25). These were then used to calculate the composite ratios NLR and PLR. PET/CT scan analysis focused on tumor ${ }^{18}$ F-FDG uptake (25).

The median age of the included patients was $61.6 \mathrm{y}$ and $58.9 \%$ were male, with $50.6 \%$ having never smoked (25). Most patients had adenocarcinomas $(76.7 \%)$ and were treated by lobectomy (87.1\%) (25). There were 144 recurrences, and the median follow up was 29.5 mo (25). Patients with a high TSUVmax had significantly higher white cell count $(P<0.001)$ and neutrophil $(P<$ $0.001)$ and lymphocyte counts $(P=0.021)$ and a greater NLR $(P=$ 0.016) (25). On univariate Cox regression analysis, white cell count $(P=0.028)$, TSUVmax $(P<0.001)$, age $(P<0.001)$, sex $(P=$ $0.003)$, smoking $(P=0.002)$, cell type $(P=0.001)$, and TNM stage $(P<0.001)$ were significantly associated with disease-specific survival (25). On multivariate analysis, TSUVmax (hazard ration $[\mathrm{HR}], 2.22$; 95\% confidence interval [CI], 1.52-3.25; $P<$ 0.001 ), tumor stage (HR, 2.11; 95\% CI, 1.47-3.01; $P<0.001$ ), and old age (HR, 1.03; 95\% CI, 1.01-1.05; $P=0.002$ ) remained independently prognostic in terms of disease-specific survival (25).

\section{DISCUSSION}

The results of the present systematic review showed that, in most of the studies, there was a direct relationship between the tumor and BM glucose uptake and host systemic inflammatory responses in patients with common solid tumors.

Both tumor and nodal glucose uptake and BM glucose uptake were associated with poor outcome in these patients. Although BM ${ }^{18}$ F-FDG accumulation may mainly reflect inflammatory 
responses, tumor and nodal ${ }^{18} \mathrm{~F}-\mathrm{FDG}$ accumulation reflect the malignant grade of the tumor cells in addition to the inflammatory responses. Therefore, it may be that the nature of their associations with survival will be different.

Taken together, the present review provides new insight into the interaction between tumor and host. This may suggest new approaches to more optimal therapeutic targeting and monitoring strategies for patients with cancer.

The basis of the relationship between tumor glucose uptake and markers of the systemic inflammatory response is not clear. The importance of the TME is increasingly appreciated. In addition to the tumor cells themselves, stromal cells and inflammatory cells are now recognized to play a role in growth and progression of cancer. The predominant cells in the tumor stroma are the cancerassociated fibroblasts that have been shown to promote tumor progression and invasion through the production of growth factors, cytokines, and metabolites and to stimulate blood vessel formation (32). Such stromal cell activity is intimately linked to inflammatory cell activity, and macrophages contribute to tumor progression and spread by the promotion of genetic instability, protection and nurturing of cancer stem cells, promotion of metastatic spread, and the downregulation of the protective T-cell driven adaptive immune response (33-35). In turn, such macrophage activity appears to be dependent on the tumor stage, tissue involvement, and microbiota (33). The macrophage influence on tumor activity can be proinflammatory and tumor-growth-promoting via the classic M1 pathway commonly upregulated by the inflammatory cytokines TNF- $\alpha$ and IL-6 (36) as well as antiinflammatory and tumorgrowth-reducing via the alternative M2 pathway commonly unpregulated by the antiinflammatory cytokines IL-4 and IL-10 (36).

The importance of neutrophil activity and infiltrate in cancer progression and metastasis has become an increasingly recognized prognostic domain. Neutrophil activity has been shown to increase tumor progression by facilitating and encouraging angiogenesis (37). Neutrophil activity has also been implicated in potentiating tumor growth through the activation of specific inflammatory cytokines, particularly IL-1 and IL-6, and via amino acid depletion (37) and promotes angiogenesis and the metastatic potential of cancer (37). Neutrophils have also been shown to direct cancer cell growth toward endothelial cells, which can lead to increased hematologic spread promoting distant metastasis (37). Indeed in the premetastatic state in patients with advanced cancer, neutrophil clusters or localized build-ups in distant organs have been shown to be predictive of eventual metastatic spread (37).

Finally, it has also been postulated that cytokines produced by the tumor/stroma complex can lead to marrow mesenchymal cell recruitment, thus providing a potential explanation for increased marrow activation seen in the present review (35).

However, there is recognized uptake of ${ }^{18} \mathrm{~F}-\mathrm{FDG}$ by both tumor and inflammatory cells and that the TME consists of both tumor and inflammatory cells (38). Therefore, part of the glucose uptake into the tumor may be due to the infiltration of inflammatory cells. Indeed, Rosenberg et al. proposed caution when analyzing PET/CT scans as the marrow hypermetabolism shown may be due to inflammation and not necessarily where the tumor cells are located (39).

Although BM mesenchymal stem cells, monocyte, or platelet progenitor cells are unregulated during the response to active malignancy, an elevation of neutrophils, which is quantitatively the most important cell type, has been consistently seen in patients with active cancer as shown by the prognostic strength of neutrophils singularly and NLR $(15,40)$.

However, confirmation of this hypothesis will require careful histologic examination of the areas of both tumor and BM increased signal uptake. Irrespective, it is clear that both tumor and inflammatory cells display signs of the Warburg effect, and it may be that both contribute to the increased lactate dehydrogenase and its prognostic value observed in patients with cancer $(41,42)$.

In the present review it was confirmed that there was a relationship between tumor and BM glucose uptake and poor outcome in patients with cancer, confirming its clinical utility. Given that 2 recent metaanalyses have established the prognostic strength of both singular and combined markers of the systemic inflammatory response in both operable and inoperable disease across multiple cancer types $(15,16)$, it remains to be determined whether the prognostic value of tumor and BM glucose uptake is determined by the systemic inflammatory response or vice versa.

Although most of the above studies used singular markers of the systemic inflammatory response these have now been surpassed by the use of composite ratios and cumulative scores $(15,16)$. Furthermore, in a recent study in operable colon cancer Dolan et al. showed that both composite ratios and cumulative scores had prognostic value, independent of TNM stage (40). However, cumulative scores, based on normal reference ranges, are simpler and more consistent for clinical use and should be used in future research to investigate the association between ${ }^{18} \mathrm{~F}$-FDG PET imaging and host inflammatory responses."

The importance of the relationship between tumor and BM glucose uptake and the systemic inflammatory response is of more than academic interest, particularly in the era of immunomodulatory therapy for patients with advanced cancer. In particular, modulation of the innate and adaptive immune responses will shed new light on the nature of this relationship (43). Furthermore, whereas there was some heterogeneity in the results, there was a relationship between tumor and BM glucose uptake and poor outcomes in 5 studies including 1,525 patients.

\section{CONCLUSION}

To our knowledge, this is the first systematic review to examine the relationship between tumor glucose metabolism using PET/CT imaging and host inflammatory responses. From the review, there appeared to be a direct relationship between the tumor and BM glucose uptake and host systemic inflammatory responses in patients with common solid tumors. Furthermore, there was a relationship between tumor and BM glucose uptake and poor outcome in these patients.

\section{DISCLOSURE}

This study was funded by the University of Glasgow. No other potential conflict of interest relevant to this article was reported.

\section{REFERENCES}

1. World Health Organization. Cancer. World Health Organization website. http:// www.who.int/mediacentre/factsheets/fs297/en/. Updated September 12, 2018. Accessed December 11, 2018.

2. Roxburgh CS, McMillan DC. Role of systemic inflammatory response in predicting survival in patients with primary operable cancer. Future Oncol. 2010;6:149-163.

3. Proctor MJ, Morrison DS, Talwar D, et al. A comparison of inflammation-based prognostic scores in patients with cancer: a Glasgow Inflammation Outcome Study. Eur J Cancer. 2011;47:2633-2641.

4. Hanahan D, Weinberg RA. The hallmarks of cancer. Cell. 2000;100:57-70.

5. Hanahan D, Weinberg RA. Hallmarks of cancer: the next generation. Cell. 2011;144: 646-674. 
6. Park JH, Powell AG, Roxburgh CS, Horgan PG, McMillan DC, Edwards J. Mismatch repair status in patients with primary operable colorectal cancer: associations with the local and systemic tumour environment. Br J Cancer. 2016; 114:562-570.

7. Balkwill FR, Capasso M, Hagemann T. The tumor microenvironment at a glance. J Cell Sci. 2012;125:5591-5596.

8. McAllister SS, Weinberg RA. The tumour-induced systemic environment as a critical regulator of cancer progression and metastasis. Nat Cell Biol. 2014;16: 717-727.

9. Warburg O. On the origin of cancer cells. Science. 1956;123:309-314.

10. Maguire D, Neytchev O, Talwar D, McMillan D, Shiels PG. Telomere homeostasis: interplay with magnesium. Int J Mol Sci. 2018;19:E157.

11. Quail DF, Joyce JA. Microenvironmental regulation of tumor progression and metastasis. Nat Med. 2013;19:1423-1437.

12. Park JH, Watt DG, Roxburgh CS, Horgan PG, McMillan DC. Colorectal cancer, systemic inflammation, and outcome: staging the tumor and staging the host. Ann Surg. 2016;263:326-336.

13. Guthrie GJ, Charles KA, Roxburgh CS, Horgan PG, McMillan DC, Clarke SJ. The systemic inflammation-based neutrophil-lymphocyte ratio: experience in patients with cancer. Crit Rev Oncol Hematol. 2013;88:218-230.

14. McMillan DC. The systemic inflammation-based Glasgow prognostic score: a decade of experience in patients with cancer. Cancer Treat Rev. 2013;39:534540.

15. Dolan RD, McSorley ST, Horgan PG, Laird B, McMillan DC. The role of the systemic inflammatory response in predicting outcomes in patients with advanced inoperable cancer: Systematic review and meta-analysis. Crit Rev Oncol Hematol. 2017;116:134-146.

16. Dolan RD, Lim J, McSorley ST, Horgan PG, McMillan DC. The role of the systemic inflammatory response in predicting outcomes in patients with operable cancer: systematic review and meta-analysis. Sci Rep. 2017;7:16717.

17. Kijima S, Sasaki T, Nagata K, Utano K, Lefor AT, Sugimoto H. Preoperative evaluation of colorectal cancer using CT colonography, MRI, and PET/CT. World J Gastroenterol. 2014;20:16964-16975.

18. Griffeth LK. Use of PET/CT scanning in cancer patients: technical and practical considerations. Proc Bayl Univ Med Cent. 2005;18:321-330.

19. Miele E, Spinelli GP, Tomao F, et al. Positron emission tomography (PET) radiotracers in oncology: utility of ${ }^{18}$ F-fluoro-deoxy-glucose (FDG)-PET in the management of patients with non-small-cell lung cancer (NSCLC). $J$ Exp Clin Cancer Res. 2008;27:52.

20. Inoue K, Goto R, Okada K, Kinomura S, Fukuda H. A bone marrow F-18 FDG uptake exceeding the liver uptake may indicate bone marrow hyperactivity. Ann Nucl Med. 2009;23:643-649.

21. Prévost S, Boucher L, Larivee P, Boileau R, Benard F. Bone marrow hypermetabolism on ${ }^{18} \mathrm{~F}$-FDG PET as a survival prognostic factor in non-small cell lung cancer. J Nucl Med. 2006;47:559-565.

22. Cicone F, Loose D, Deron P, et al. Prognostic value of FDG uptake by the bone marrow in squamous cell carcinoma of the head and neck. Nucl Med Commun. 2008;29:431-435.

23. Chang PY, Kuo YB, Wu TL, et al. Association and prognostic value of serum inflammation markers in patients with leukoplakia and oral cavity cancer. Clin Chem Lab Med. 2013;51:1291-1300.

24. Chen HH, Wang HM, Fan KH, et al. Pre-treatment levels of C-reactive protein and squamous cell carcinoma antigen for predicting the aggressiveness of pharyngolaryngeal carcinoma. PLoS One. 2013;8:e55327.
25. Jeong E, Hyun SH, Moon SH, Cho YS, Kim BT, Lee KH. Relation between tumor FDG uptake and hematologic prognostic indicators in stage I lung cancer patients following curative resection. Medicine (Baltimore). 2017;96:e5935.

26. Zhong L, Li C, Ren Y, Wu D. Prognostic value of ${ }^{18}$ F-fluorodeoxyglucose PET parameters and inflammation in patients with nasopharyngeal carcinoma. Oncol Lett. 2017;14:5004-5012.

27. Lee JW, Na JO, Kang DY, Lee SY, Lee SM. Prognostic significance of FDG uptake of bone marrow on PET/CT in patients with non-small-cell lung cancer after curative surgical resection. Clin Lung Cancer. 2017;18:198-206.

28. Lee JW, Seo KH, Kim ES, Lee SM. The role of ${ }^{18} \mathrm{~F}$-fluorodeoxyglucose uptake of bone marrow on PET/CT in predicting clinical outcomes in non-small cell lung cancer patients treated with chemoradiotherapy. Eur Radiol. 2017;27:1912-1921.

29. Lee JW, Lee MS, Chung IK, Son MW, Cho YS, Lee SM. Clinical implication of FDG uptake of bone marrow on PET/CT in gastric cancer patients with surgical resection. World J Gastroenterol. 2017;23:2385-2395.

30. McSorley ST, Khor BY, Tsang K, et al. The relationship between ${ }^{18} \mathrm{~F}$ FDGPETCT derived markers of tumour metabolism and systemic inflammation in patients with recurrent disease following surgery for colorectal cancer. Colorectal Dis. 2018;20:407-415.

31. Lee JW, Baek MJ, Ahn TS, Lee SM. Fluorine-18-fluorodeoxyglucose uptake of bone marrow on PET/CT can predict prognosis in patients with colorectal cancer after curative surgical resection. Eur J Gastroenterol Hepatol. 2018;30:187-194.

32. van Pelt GW, Sandberg TP, Morreau H, et al. The tumour-stroma ratio in colon cancer: the biological role and its prognostic impact. Histopathology. 2018;73: 197-206.

33. Mantovani A, Marchesi F, Malesci A, Laghi L, Allavena P. Tumour-associated macrophages as treatment targets in oncology. Nat Rev Clin Oncol. 2017;14:399-416.

34. Mantovani A, Romero P, Palucka AK, Marincola FM. Tumour immunity: effector response to tumour and role of the microenvironment. Lancet. 2008;371:771-783.

35. Kudo-Saito C. Cancer-associated mesenchymal stem cells aggravate tumor progression. Front Cell Dev Biol. 2015;3:23.

36. Martinez FO, Gordon S. The M1 and M2 paradigm of macrophage activation: time for reassessment. F1000Prime Rep. 2014;6:13.

37. Coffelt SB, Wellenstein MD, de Visser KE. Neutrophils in cancer: neutral no more. Nat Rev Cancer. 2016;16:431.

38. Shreve PD. Focal fluorine-18 fluorodeoxyglucose accumulation in inflammatory pancreatic disease. Eur J Nucl Med. 1998;25:259-264.

39. Rosenberg R, Herrmann K, Gertler R, et al. The predictive value of metabolic response to preoperative radiochemotherapy in locally advanced rectal cancer measured by PET/CT. Int J Colorectal Dis. 2009;24:191-200.

40. Dolan RD, McSorley ST, Park JH, et al. The prognostic value of systemic inflammation in patients undergoing surgery for colon cancer: comparison of composite ratios and cumulative scores. Br J Cancer. 2018;119:40-51.

41. Mezquita L, Auclin E, Ferrara R, et al. Association of the lung immune prognostic index with immune checkpoint inhibitor outcomes in patients with advanced non-small cell lung cancer. JAMA Oncol. 2018;4:351-357.

42. Uneno Y, Taneishi K, Kanai M, et al. Development and validation of a set of six adaptable prognosis prediction (SAP) models based on time-series real-world big data analysis for patients with cancer receiving chemotherapy: a multicenter case crossover study. PLoS One. 2017;12:e0183291.

43. Ferris RL, Lenz HJ, Trotta AM, et al. Rationale for combination of therapeutic antibodies targeting tumor cells and immune checkpoint receptors: Harnessing innate and adaptive immunity through IgG1 isotype immune effector stimulation. Cancer Treat Rev. 2018;63:48-60. 